\title{
Wilson-Fisher fixed points for any dimension
}

\author{
R. Trinchero \\ Instituto Balseiro, Centro Atómico Bariloche, 8400 San Carlos de Bariloche, Argentina and CONICET, \\ Rivadavia 1917, 1033 Capital Federal, Argentina
}

(Received 5 April 2019; published 4 December 2019)

\begin{abstract}
The critical behavior of a nonlocal scalar field theory is studied. This theory has a nonlocal quartic interaction term which involves a power $-\beta$ of the Laplacian. The power $-\beta$ is tuned so as to make that interaction marginal for any dimension. This leads to integer or half-integer values for $\beta$, depending on the space dimension. Introducing an auxiliary field, it is shown that the theory can be renormalized by means of local counterterms in the fields. The lowest order Feynman diagrams corresponding to coupling constant renormalization, mass renormalization, and field renormalization are computed. In all cases, a nontrivial IR fixed point is obtained. Remarkably, for dimensions other than 4, field renormalization is required at the one-loop level. For $d=4$, the theory reduces to the usual local $\phi^{4}$ field theory, and field renormalization is required starting at the two-loop level. The critical exponents $\nu$ and $\eta$ are computed for dimensions $2,3,4$, and 5. For dimensions greater than 4 , the critical exponent $\eta$ turns out to be negative for $\epsilon>0$, which indicates a violation of the unitarity bounds.
\end{abstract}

DOI: 10.1103/PhysRevD.100.116004

\section{INTRODUCTION}

The computation of critical exponents for the threedimensional Ising model using the $\epsilon$-expansion provides a concrete example of the relevance of the renormalization group ideas [1,2]. This is done by considering a selfinteracting $\phi^{4}$ theory in $d=4-\epsilon$ dimensions, where $\epsilon$ is allowed to take real values. This procedure leads to a qualitative understanding of the three-dimensional Ising model physics, and to predictions for critical exponents in reasonable agreement with the exact values.

The renormalization group consists of the study of the evolution of a system under scale transformations. This system involves all possible interactions of any range for all kinds of dynamical variables. Different physical systems correspond to the study of particular fixed points in this huge space of couplings. This paper studies a particular example of a system described near the corresponding fixed point by a nonlocal field theory. Nonlocal field theories appear in various aspects of physics. These include proposals for dealing with quantum gravity [3], field theories based on noncommutative geometry [4], and critical phenomena. The use of nonlocal field theories in the description of critical phenomena is not new [5-9]. Such models appear in statistical systems with long-range interactions. In a previous paper [10], the critical behavior of a $\phi^{4}$ theory with

Published by the American Physical Society under the terms of the Creative Commons Attribution 4.0 International license. Further distribution of this work must maintain attribution to the author(s) and the published article's title, journal citation, and DOI. Funded by SCOAP ${ }^{3}$. a nonlocal kinetic term was studied. That theory has asymptotic freedom in the UV. In this paper, the critical behavior of a field theory which includes a nonlocal interaction is studied. This interaction consists of a $\phi^{4}$ term which involves a power $-\beta$ of the Laplacian. The parameter $\beta$ allows one to tune the dimensions of the corresponding coupling constant. This is done in such a way that for any space dimension $n$ the coupling is adimensional, thus reproducing for any spatial dimension $n$ what happens for the local $\phi^{4}$ theory in $n=4$ dimensions. This last feature is the main motivation to consider that interaction in this work. The theory is formulated using an auxiliary field $\rho$, which renders the interaction local, at the cost of having a nonlocal kinetic term. The renormalization and critical properties of this theory are studied at the one-loop level. The features and results of this work are summarized as follows:

(a) The theory to be considered is a scalar field theory involving a nonlocal $\phi^{4}$ interaction term which includes a power $-\beta$ of the Laplacian. This power is chosen so as to make the coupling marginal for any dimension.

(b) By means of an auxiliary field $\rho$ with a nonstandard kinetic term, the quartic nonlocal interaction can be replaced by a local cubic interaction. It is shown that the effective action for the theory in terms of the two fields $\rho$ and $\phi$ can be renormalized by means of local counterterms in the fields $\rho$ and $\phi$. The structure of these counterterms is described for all values of $n$.

(c) The theory is dimensionally regularized, and the contribution of the one-loop Feynman diagrams which present poles when $\epsilon \rightarrow 0$ is computed.

(d) These one-loop computations show that mass and coupling constant renormalization are required at this 
level in order to render the theory finite. In addition, and contrasting with the local $\phi^{4}$ theory, field renormalization is required at this one-loop level for any dimension other than 4.

(e) The previous calculation allows one to compute the fixed point value for the coupling constant, and the critical exponents $\nu$ and $\eta$. In all cases, a nontrivial IR fixed point is obtained for $\epsilon>0$.

(f) For dimensions $d=n-\epsilon$ with $n$ less than 4, nontrivial fixed points are obtained describing consistent quantum theories.

(g) For dimensions $d=n-\epsilon$ with $n$ greater than 4 , the critical exponent $\eta$ turns out to be negative for $\epsilon>0$, which indicates a violation of the unitarity bounds.

The paper is organized as follows. Section II presents the model and explains how to tune $\beta$ in order to get an adimensional coupling. Section III presents the renormalization procedure to be employed. Section IV contains the computations of Feynman diagrams. Section IVA presents the one-loop corrections to the two-point function, which involve the contribution of two diagrams. Section IV B deals with the one-loop corrections to the four-point function, which involve the contribution of three diagrams. Section IV C computes the critical exponents $\nu$ and $\eta$ for these theories. Section V deals with the unitarity bounds that are obtained when assuming that the theory provides a unitary representation of the conformal group. Finally, Sec. VI presents some concluding remarks and additional research motivated by this work. An appendix describing the structure of counterterms for each $n$ is also included.

\section{THE ACTION}

The Euclidean action for the field $\phi$ to be considered is given by

$$
\begin{aligned}
& \tilde{S}=\tilde{S}_{0}+\tilde{S}_{I}, \quad \tilde{S}_{0}=\int d^{n} x \frac{1}{2} \phi\left(-\triangle+m_{0}^{2}\right) \phi, \\
& \tilde{S}_{I}=\frac{\lambda_{0}}{4 !} \int d^{d} x \phi^{2}\left(-\triangle+M^{2}\right)^{-\beta} \phi^{2} .
\end{aligned}
$$

In what follows, bare mass and coupling will be indicated by $m_{0}$ and $\lambda_{0}$, and the corresponding renormalized quantities will be $m$ and $\lambda$. Using an auxiliary field $\rho$, the action $\tilde{S}$ can be replaced by the following equivalent action,

$$
\begin{aligned}
S & =S_{0}+S_{I}, \\
S_{0} & =\int d^{n} x\left[\frac{1}{2} \phi\left(-\triangle+m_{0}^{2}\right) \phi-\frac{1}{2} \rho\left(-\triangle+M^{2}\right)^{\beta} \rho\right], \\
S_{I} & =-\int d^{n} x \sqrt{\frac{\lambda_{0}}{12}} \rho \phi^{2} .
\end{aligned}
$$

Functionally integrating over $\rho$ leads back to the action $\tilde{S}$, i.e.,

$$
\int \mathcal{D} \rho e^{-S} \propto e^{-\tilde{S}}
$$

The free energy is given by

$$
F=-\log Z, \quad Z=\int \mathcal{D} \phi \mathcal{D} \rho e^{-S} .
$$

The Fourier transforms of the free $\rho$ and $\phi$ two-point functions are therefore given by

$$
\langle\rho \rho\rangle(p)=-\frac{1}{\left(p^{2}+M^{2}\right)^{\beta}}, \quad\langle\phi \phi\rangle(p)=\frac{1}{\left(p^{2}+m_{0}^{2}\right)} ;
$$

the mass $M$ has been included in order to regulate infrared divergencies.

It is noted that the dimensions of the field $\phi$ and coupling $\left[\lambda_{0}\right]$ are

$$
[\phi]=\frac{d-2}{2}, \quad\left[\lambda_{0}\right]=d-4[\phi]+2 \beta=4-d+2 \beta .
$$

For $d=n-\epsilon$, choosing

$$
\beta=\frac{n}{2}-2
$$

makes the coupling dimension equal to $\epsilon$, i.e., $\left[\lambda_{0}\right]=\epsilon$. This choice will be adopted from now on. This is exactly what happens for the coupling of the local $\phi^{4}$ theory in $d=4-\epsilon$. Thus the nonlocal coupling parametrized by $\beta$ in $\tilde{S}_{I}$ can be considered as a device to reproduce the same situation in other dimensions. ${ }^{1}$ It is also noted that if $n>4$, then $\beta>0$, which for $M \rightarrow 0$ produces infrared divergencies in these cases. This motivates the choice $M=M_{n}$, where

$$
M_{n}=\left\{\begin{array}{ll}
0 & n \leq 4 \\
M & n>4
\end{array} .\right.
$$

\section{RENORMALIZATION PROCEDURE}

\section{A. Degree of divergence}

The degree of divergence for a proper or 1PI graph $G$ with $E_{\phi}$ external $\phi$ lines and $E_{\rho}$ external $\rho$ lines is given by

$$
\omega(G)=n-E_{\phi}\left(\frac{n-2}{2}\right)-2 E_{\rho} .
$$

There is no dependence on the number of vertices because the coupling $\lambda$ is adimensional for any $n$, thanks to the choice of Eq. (2.3). Requiring the quantum theory to respect the symmetry of the classical action under $\phi \rightarrow-\phi$ implies that a graph with $E_{\phi}$ odd should vanish. Thus $E_{\phi}$ is

\footnotetext{
${ }^{1}$ Marginality can also be attained by changing the power in the interaction and the number of derivatives in the kinetic term. This can be done without considering nonlocal terms, see [11] and the references therein.
} 
taken to be even. Therefore $\omega(G)$ is always a integer. Thus taking a derivative respect to a external momenta of a given proper graph would lower its degree of divergence by 1 . This implies that when $\omega(G) \geq 0$, the divergent part of the proper diagrams will be given by polynomials in the external momenta of order $\omega(G)$, if $\omega(G)<0$, there is no divergent part. Thus only local counterterms in the fields $\phi$ and $\rho$ and its derivatives are required to renormalize any proper graph for the theory described by the action (2.2). The explicit computations appearing in the next section are examples of this fact. The explicit form of counterterms depending on the dimension $n$ is given in the Appendix.

\section{B. Effective theory for the $\phi$ field}

This effective theory is described by the effective action $\Gamma_{\phi}$ derived from the functional integral, which involves the action (2.1) - that is, without a dependence on the auxiliary field $\rho$. In terms of the diagrams obtained from the perturbative expansion of the functional integral written using the action (2.2) which involves $\rho$ lines, the diagrams contributing to the effective action $\Gamma_{\phi}$ are obtained from the former ones by collapsing $\rho$ lines and including the nonlocal four leg vertex described by $\tilde{S}_{I}$. Therefore the relevant diagrams for $\Gamma_{\phi}$ include diagrams which are not 1PI for the $\rho$ lines, i.e., that can be divided into two disconnected parts by cutting a $\rho$ line. The diagrams appearing in subsections 4.2.1 and 4.2.2 are Figs. 2 and 3 are examples of graphs that are not 1PI for the $\rho$ lines but instead 1PI for the $\phi$ lines. It is clear that since, as shown in the previous section, the theory described by $S_{I}$ can be renormalized by means of local counterterms in the fields $\phi$ and $\rho$ and its derivatives, then the theory described by $\tilde{S}_{I}$ can also be renormalized. However, since the $\rho$ lines are, in general, nonlocal, then the counterterms required for renormalizing the effective action $\Gamma_{\phi}$, can be nonlocal. This nonlocality is harmless since it is totally under control at the level of the effective action involving the two fields $\phi$ and $\rho$, from which the effective action involving only the $\phi$ field can be derived.

\section{EFFECTIVE COUPLINGS}

\section{A. One-loop correction to the two-point function}

The one-loop corrections to the two-point function are given by the following diagrams.

It is noted that the internal $\rho$ line in the first diagram carries zero momentum. Thus, taking into account the relation (2.3), this line gives a factor

$$
\frac{-1}{M_{n}^{2 \beta}}=\frac{-1}{M_{n}^{n-4}},
$$

which, recalling Eq. (2.4), shows that this line vanishes for $n<4$, gives -1 for $n=4$, and is nonvanishing for $n>4$.

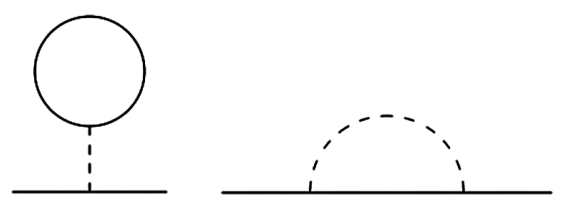

FIG. 1. Lowest order diagrams contributing to renormalization of the $\phi$ two-point function.

Indeed, the motivation for introducing the mass $M_{n}$ for the $\rho$ field was to regulate the infrared divergence appearing from this propagator at zero momentum for $n>4$, i.e., $\beta>0$. The contribution of the first diagram without external legs is

$$
\begin{aligned}
a_{T}(d, \beta) & =\mu_{T} \frac{-\lambda_{0}}{2 ! 12} M_{n}^{4-n} I_{T}(d)=\frac{-\lambda_{0}}{6} M_{n}^{4-n} I_{T}(d), \\
I_{T}(d) & =\frac{d^{d} q}{(2 \pi)^{d}} \frac{1}{\left(q^{2}+m_{0}^{2}\right)} ;
\end{aligned}
$$

the quantity $\mu_{T}=4$ is the multiplicity of this diagram. Introducing the Feynman parametrization and integrating over the momenta $q$ leads to

$$
I_{T}(d)=\frac{\Gamma\left(1-\frac{d}{2}\right)}{(4 \pi)^{\frac{d}{2}}} m_{0}^{2\left(\frac{d}{2}-1\right)}
$$

Substituting in Eq. (4.1) gives

$$
\begin{aligned}
a_{T}(d, \beta) & =-\frac{\lambda_{0}}{6} M_{n}^{4-n} \frac{\Gamma\left(1-\frac{d}{2}\right)}{(4 \pi)^{\frac{d}{2}}} m_{0}^{2\left(\frac{d}{2}-1\right)} \\
& =-\frac{g_{0}}{6} \mu^{2(\beta+2)-d} M_{n}^{4-n} \frac{\Gamma\left(1-\frac{d}{2}\right)}{(4 \pi)^{\frac{d}{2}}} m_{0}^{2\left(\frac{d}{2}-1\right)},
\end{aligned}
$$

where in the second equality the result has been expressed in terms of the adimensional coupling $g_{0}=\mu^{d-2(\beta+2)} \lambda_{0}$. For $n$ odd, this contribution presents no divergence for $\epsilon \rightarrow 0$. For $d=n-\epsilon$, with $n$ being a positive even integer, the gamma function can be expanded as follows,

$$
\Gamma\left(1-\frac{d}{2}\right)=\Gamma\left(1-\frac{d}{2}\right)=\frac{(-1)^{\frac{n}{2}-1} 2}{\left(\frac{n}{2}-1\right) !} \frac{2}{\epsilon}+R
$$

where $R$ denotes terms that present no singularities when $\epsilon \rightarrow 0$. This leads to

$$
a_{T}(n-\epsilon, \beta)=-\frac{g_{0}}{6} \mu^{2(\beta+2)-n+\epsilon} M_{n}^{4-n} \frac{m_{0}^{2\left(\frac{n}{2}-1-\frac{\epsilon}{2}\right)}(-1)^{\frac{n}{2}-1} 2}{(4 \pi)^{\frac{d}{2}}} \frac{2}{\left(\frac{n}{2}-1\right) ! \epsilon}+R^{\prime}
$$

for $n=2$ and 3. Because of the remarks at the beginning of this subsection, this diagram gives no contribution, which is also the case for any odd $n$. Thus, for $n<6$, the singular part of $a_{T}$ is given by 


$$
a_{T}^{S P}\left(n-\epsilon, \frac{n}{2}-2\right)=\left\{\begin{array}{ll}
0 & n=2 \\
0 & n=3 \\
\frac{g_{0}}{3} \frac{m_{0}^{2}}{(4 \pi)^{2}} \frac{1}{\epsilon} & n=4 \\
0 & n=5
\end{array} .\right.
$$

The integral corresponding to the second diagram is

$$
\begin{aligned}
\Pi\left(p, m_{0}, d, \beta\right) & =-\mu_{\Pi} \frac{\lambda_{0}}{2 ! 12} I_{F}\left(p, m_{0}, d, \beta\right) \\
& =-\frac{\lambda_{0}}{3} I_{F}\left(p, m_{0}, d, \beta\right), \\
I_{F}\left(p, m_{0}, d, \beta\right) & =\int \frac{d^{d} q}{(2 \pi)^{d}} \frac{1}{\left(q^{2}+m_{0}^{2}\right)\left((p-q)^{2}+M_{n}^{2}\right)^{\beta}} ;
\end{aligned}
$$

the quantity $\mu_{\Pi}=8$ is the multiplicity of this diagram. The degree of divergence of this integral is

$$
\omega\left(I_{F}\right)=d-2-2 \beta .
$$

For the cases considered in this work $(d=n-\epsilon$, $\left.\beta=\frac{n}{2}-2\right)$, this gives $\omega\left(I_{F}\right)=2-\epsilon$. This implies that there is a divergent term proportional to $p^{2}$, i.e., that this diagram requires a field renormalization. Below it is shown that the coefficient of $p^{2}$ vanishes for $d=4$. This should be so because, for $n=4, \beta=0$ and the theory coincides with the usual local $\phi^{4}$ field theory, which requires no field renormalization at the one-loop level. Introducing the Feynman parametrization and integrating over the momenta $q$ leads to

$$
I_{F}\left(p, m_{0}, d, \beta\right)=\frac{\mu^{2\left(-\beta+\frac{d}{2}-1\right)}}{(4 \pi)^{\frac{d}{2}}} \frac{\Gamma\left(-\frac{d}{2}+\beta+1\right)}{\Gamma(\beta)} \int_{0}^{1} d x(1-x)^{\beta-1}\left(\frac{M_{n}^{2}}{\mu^{2}}(1-x)+\frac{m_{0}^{2}}{\mu^{2}} x+\frac{p^{2}}{\mu^{2}}(1-x) x\right)^{-\beta+\frac{d}{2}-1} .
$$

The power of the parenthesis in the integrand is $-\beta+\frac{d}{2}-1=1-\frac{\epsilon}{2}$, which therefore presents no singularities in the integration region. Thus $\epsilon=0$ is taken in this power. The integral can be evaluated, leading to

$$
I_{F}\left(p, m_{0}, d, \beta\right)=\frac{\mu^{-\epsilon}}{(4 \pi)^{\frac{d}{2}}} \frac{\Gamma\left(-\frac{d}{2}+\beta+1\right)}{\Gamma(\beta)} \frac{\left(\beta\left((\beta+2) M_{n}^{2}+p^{2}\right)+(\beta+2) m_{0}^{2}\right)}{\beta(\beta+1)(\beta+2)},
$$

which leads to the following expression for its singular part:

$$
I_{F}^{S P}\left(p, m_{0}, d, \beta\right)=-\frac{2^{2-n} \pi^{-n / 2}\left(M^{2} n^{2}-4 M^{2} n+2 m_{0}^{2} n+2 n p^{2}-8 p^{2}\right)}{(n-4)(n-2) n \Gamma\left(\frac{n}{2}-2\right) \epsilon} .
$$

This leads to

$$
\Pi^{S P}\left(p, m_{0}, d, \beta\right)=-\frac{g_{0}}{3} I_{F}^{S P}\left(p, m_{0}, d, \beta\right)=\left\{\begin{array}{ll}
\frac{g_{0}}{4 \pi} \frac{1}{3}\left(\frac{2}{\epsilon}\right)\left(m_{0}^{2}-p^{2}\right) & n=2 \\
\frac{g_{0}}{8 \pi^{2}} \frac{1}{9}\left(\frac{2}{\epsilon}\right)\left(6 m_{0}^{2}-2 p^{2}\right) & n=3 \\
\frac{g_{0}}{(4 \pi)^{2}} \frac{1}{3}\left(\frac{2}{\epsilon}\right) m_{0}^{2} & n=4 \\
\frac{g_{0}}{\pi^{3}} \frac{\left(5 M^{2}+2\left(5 m_{0}^{2}+p^{2}\right)\right)}{360} \frac{1}{\epsilon} & n=5
\end{array} .\right.
$$

This result is quite remarkable because it shows that, for $n \neq 4 \Rightarrow \beta \neq 0$, field renormalization is required at the one-loop level to absorb the divergences. It is recalled that for the $n=4$ local $\phi^{4}$ theory, field renormalization is first required at two loops. The diagram contributing to field renormalization is the second one in Fig. 1, and it requires field renormalization because of the nonzero momentum carried by the auxiliary field $\rho$ line. Alternatively this can be seen from the fact that the nonlocal interaction introduces momentum at each nonlocal vertex. It is remarked that the general result (4.2) shows that, starting from $n=5$, the sign of the term proportional to $p^{2}$ changes. This sign change will affect the sign of the critical exponent $\eta$, leading to a violation of the unitarity bounds for $n \geq 5$.
The effective two-point function to one-loop order is ${ }^{2}$

$$
\begin{aligned}
\Gamma_{2}(p) & =p^{2}+m_{0}^{2}-\left(a_{T}^{S P}+\Pi^{S P}\right) \\
& =p^{2} Z_{\phi}^{-1}+m_{0}^{2} Z_{m^{2}}^{-1}=Z_{\phi}^{-1}\left(p^{2}+m_{0}^{2} \frac{Z_{\phi}}{Z_{m^{2}}}\right)
\end{aligned}
$$

${ }^{2}$ The free energy is

$$
F=-\log Z, \quad Z=\int \mathcal{D} \phi \mathcal{D} \rho e^{-S},
$$

therefore the order 0 free energy goes as $S_{0}$, and the corrections carry a minus sign in front. 
where

$$
Z_{\phi}=1-\frac{g_{0}}{(4 \pi)^{\frac{n}{2}}} \frac{1}{\epsilon} c_{\phi}(n), \quad Z_{m^{2}}=1+\frac{g_{0}}{(4 \pi)^{\frac{n}{2}}} \frac{1}{\epsilon} c_{m^{2}}(n),
$$

with

$$
\begin{aligned}
& c_{\phi}(n)= \begin{cases}\frac{2}{3} & n=2 \\
\frac{4}{9} \frac{1}{\sqrt{\pi}} & n=3 \\
0 & n=4 \\
-\frac{32}{180} \frac{1}{\sqrt{\pi}} & n=5\end{cases} \\
& c_{m^{2}}(n)= \begin{cases}\frac{2}{3} & n=2 \\
\frac{4}{3 \sqrt{\pi}} & n=3 \\
1 & n=4 \\
\frac{32}{36 \sqrt{\pi}}\left(1+\frac{M_{n}^{2}}{2 m_{0}^{2}}\right) & n=5\end{cases}
\end{aligned}
$$

The effective mass is therefore given $b^{3}$

$$
m^{2}=m_{0}^{2} \frac{Z_{\phi}}{Z_{m^{2}}}
$$

The gamma function is given by

$$
\begin{aligned}
\gamma(g) & =\left.\mu \frac{\partial}{\partial \mu} \log Z_{\phi}^{\frac{1}{2}}\right|_{\lambda \text { fixed }} \\
& =\frac{1}{2} \frac{\partial}{\partial \log \mu} \log \left(1-\frac{\lambda_{0} \mu^{-\epsilon}}{(4 \pi)^{\frac{n}{2}}}-c_{\phi}(n)\right) \\
& =\frac{1}{2 Z_{\phi}}\left(\frac{g_{0}}{(4 \pi)^{\frac{n}{2}}} c_{\phi}(n)\right)=\frac{1}{2} \frac{g_{0}}{(4 \pi)^{\frac{n}{2}}} c_{\phi}(n) .
\end{aligned}
$$

Thus

$$
\gamma(g)= \begin{cases}\frac{1}{3} \frac{g_{0}}{4 \pi} & n=2 \\ \frac{2}{9} \frac{1}{\sqrt{\pi}} \frac{g_{0}}{(4 \pi)^{\frac{3}{2}}} & n=3 \\ 0 & n=4 \\ -\frac{16}{180 \sqrt{\pi}} \frac{g_{0}}{(4 \pi)^{\frac{5}{2}}} & n=5\end{cases}
$$

\footnotetext{
${ }^{3}$ It is noted that, for $d=4-\epsilon$, the expression for the effective mass is

$$
m^{2}=m_{0}^{2}-\frac{g_{0} m_{0}^{2}}{(4 \pi)^{2}} \frac{1}{\epsilon}\left(\frac{1}{3}+\frac{2}{3}\right)=m_{0}^{2}-\frac{g_{0} m_{0}^{2}}{(4 \pi)^{2}} \frac{1}{\epsilon},
$$

which is the well-known result for the local $\phi^{4}$ theory.
}

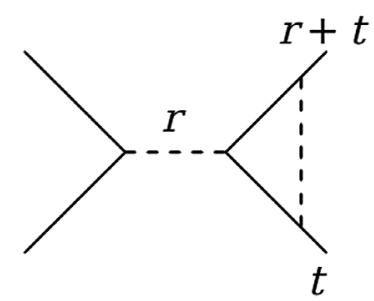

FIG. 2. $\rho \phi^{2}$ vertex correction contributing to the four-point $\phi$ function.

\section{B. One-loop correction to the four-point function \\ 1. Vertex correction}

The diagram to be considered is given by Fig. 2 .

Removing external legs, the integral to be computed is

$$
\begin{aligned}
& I_{V}\left(r, t, m_{0}, M_{n}, d, \beta\right) \\
& \quad=\frac{\mu_{V}}{4 !}\left(\frac{\lambda_{0}}{12}\right)^{2}\left(r^{2}+M_{n}^{2}\right)^{-\beta} A_{V}\left(r, t, m_{0}, M_{n}, d, \beta\right) \\
& \quad=\frac{2}{3} \lambda_{0}^{2}\left(r^{2}+M_{n}^{2}\right)^{-\beta} A_{V}\left(r, t, m_{0}, M_{n}, d, \beta\right), \\
& A_{V}\left(r, t, m_{0}, M_{n}, d, \beta\right) \\
& \quad=\int \frac{d^{d} q}{(2 \pi)^{d}} \frac{1}{\left(q^{2}+m_{0}^{2}\right)\left((q-r)^{2}+m_{0}^{2}\right)\left((q-r-t)^{2}+M_{n}^{2}\right)^{\beta}} ;
\end{aligned}
$$

the quantity $\mu_{V}=964$ ! is the multiplicity of this diagram. The overall factor $\frac{2}{3}$ in front of this contribution comes from

$$
\frac{\mu_{V}}{4 ! 12^{2}}=\frac{2}{3} \text {. }
$$

The degree of divergence of this integral is

$$
\omega\left(A_{V}\right)=d-4-2 \beta .
$$

For the cases considered in this work $(d=n-\epsilon$, $\left.\beta=\frac{n}{2}-2\right)$, this gives $\omega\left(A_{V}\right)=d-4-2 \beta=-\epsilon$. Therefore taking derivatives with respect to $M_{n}^{2}$ makes the integral convergent. Thus, making a series expansion in powers of $M_{n}^{2}$, only the first term can produce a divergence when $\epsilon \rightarrow 0$; i.e., $M_{n}=0$ is taken in the calculation below. The same reasoning applies for the dependence of $A_{V}(r, t$, $\left.m_{0}, M_{n}, d, \beta\right)$ on the external momenta $r$ and $t$, thus only zero external momenta is relevant for the singular part of this contribution. This leads to

$A_{V}\left(0,0, m_{0}, 0, d, \beta\right)=\int \frac{d^{d} q}{(2 \pi)^{d}} \frac{1}{\left(q^{2}+m_{0}^{2}\right)\left(q^{2}+m_{0}^{2}\right)\left(q^{2}\right)^{\beta}}$.

Introducing the Feynman parametrization and integrating over the moment $q$ leads to 


$$
\begin{aligned}
& A_{V}\left(0,0, m_{0}, 0, d, \beta\right) \\
& =\frac{\Gamma\left(-\frac{d}{2}+\beta+2\right)}{(4 \pi)^{\frac{d}{2}} \Gamma(\beta)} \int_{0}^{1} d y y^{\beta-1}(1-y)\left(m_{0}^{2}(1-y)\right)^{\frac{1}{2}(d-2(\beta+2))} \\
& =\frac{\Gamma\left(-\frac{d}{2}+\beta+2\right)}{(4 \pi)^{\frac{d}{2}} \Gamma(\beta)} \int_{0}^{1} d y y^{\beta-1}(1-y)^{1+\frac{1}{2}(d-2(\beta+2))} m_{0}^{d-2(\beta+2)} \\
& =\frac{\Gamma\left(\frac{d}{2}-\beta\right) \Gamma\left(-\frac{d}{2}+\beta+2\right)}{(4 \pi)^{\frac{d}{2}} \Gamma\left(\frac{d}{2}\right)} \mu^{d-2(\beta+2)}\left(\frac{m_{0}}{\mu}\right)^{d-2(\beta+2)} \\
& \stackrel{d=n-\epsilon, \beta=\frac{n}{2}-2 \Gamma\left(2-\frac{\epsilon}{2}\right) \Gamma\left(\frac{\epsilon}{2}\right)}{(4 \pi)^{\frac{d}{2}} \Gamma\left(\frac{n-\epsilon}{2}\right)} \mu^{-\epsilon}\left(\frac{m_{0}}{\mu}\right)^{-\epsilon},
\end{aligned}
$$

whose pole term in $\epsilon$ is

$$
A_{V}\left(0,0, m_{0}, 0, d, \beta\right)=\frac{\mu^{-\epsilon}}{(4 \pi)^{\frac{d}{2}}} \frac{2}{\Gamma\left(\frac{n}{2}\right)} \frac{1}{\epsilon},
$$

leading to

$$
I_{V}\left(r, t, m_{0}, M, d, \beta\right)=\frac{2}{3} \lambda_{0}^{2}\left(r^{2}+M^{2}\right)^{-\beta} \frac{\mu^{-\epsilon}}{(4 \pi)^{\frac{d}{2}}} \frac{2}{\Gamma\left(\frac{n}{2}\right)} \frac{1}{\epsilon} .
$$

\section{2. $\rho$ self-energy correction}

The diagram to be considered is shown in Fig. 3.

The integral to be computed is

$$
\begin{aligned}
I_{\rho}\left(p, m_{0}, d\right) & =\frac{\mu_{\rho}}{4 !}\left(\frac{\lambda_{0}}{12}\right)^{2} \frac{1}{\left(p^{2}+M^{2}\right)^{2 \beta}} A\left(p, m_{0}, d\right) \\
& =\frac{2}{3} \lambda_{0}^{2}\left(p^{2}+M^{2}\right)^{-2 \beta} A\left(p, m_{0}, d\right), \\
A\left(p, m_{0}, d\right) & =\int \frac{d^{d} q}{(2 \pi)^{d}} \frac{1}{\left(q^{2}+m_{0}^{2}\right)\left((p-q)^{2}+m_{0}^{2}\right)} .
\end{aligned}
$$

For this diagram, $\mu_{\rho}=964$ !, leading to the same overall factor, $\frac{2}{3}$, as in the previous diagram. Introducing the Feynman parametrization and integrating over $q$ leads to

$$
A\left(p, m_{0}, d\right)=\frac{\Gamma\left(2-\frac{d}{2}\right)}{(4 \pi)^{\frac{n}{2}}} \int_{0}^{1} d x \frac{1}{\left(m_{0}^{2}+p^{2} x(1-x)\right)^{2-\frac{d}{2}}} .
$$

The integral over $x$ gives a finite result for any $n$ since the integrand is well behaved in the entire integration region. Therefore $\Gamma\left(2-\frac{d}{2}\right)$ is the one that can produce a pole for $\epsilon \rightarrow 0$; this does not happen for $d=n-\epsilon$ with $n$ odd, nor for $n=2$. Thus only even values of $n$ with $n \geq 4$ produce

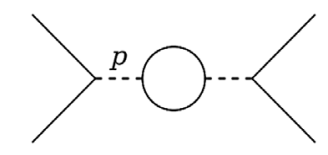

FIG. 3. $\rho$ self-energy correction contributing to the four-point $\phi$ function. poles. For $n=4$, the original integral is logarithmically divergent, and therefore, in getting the pole term, only the value of the integral for $p^{2}=0$ should be computed. This gives

$$
A\left(p, m_{0}, 4-\epsilon\right)=\frac{\Gamma\left(\frac{\epsilon}{2}\right)}{(4 \pi)^{\frac{n}{2}}}\left(m_{0}^{2}\right)^{-\frac{\epsilon}{2}}+C^{\prime \prime}=\frac{\left(m_{0}^{2}\right)^{-\frac{\epsilon}{2}}}{(4 \pi)^{\frac{n}{2}}} \frac{2}{\epsilon}+C^{\prime \prime \prime} .
$$

For $n \geq 6$ even, the integral $A\left(p, m_{0}, d\right)$ has a degree of divergence greater than zero, given by $\omega(A)=n-\epsilon-4$. This means that the derivatives of $A\left(p, m_{0}, d\right)$ with respect to $p^{2}$ have divergent coefficients when $\epsilon \rightarrow 0$ up to order $\omega(A) / 2$. The number of these divergent coefficients matches the number of parameters needed to specify the $\rho$ free field action. Indeed, for $n=6, \beta=1$, which means that the coefficient of $\rho^{2}$ and the coefficient of $\rho \triangle \rho$ get renormalized in this case. The cases with $n \geq 6$ even will not be further studied in this work.

Thus for $n<6$, the following result for the pole term of $I_{\rho}\left(p, m_{0}, d\right)$ is obtained,

$$
I_{\rho}\left(p, m_{0}, d\right)=\delta_{n, 4} \frac{2}{3} \lambda_{0}^{2} \frac{\mu^{-\epsilon}}{(4 \pi)^{\frac{n}{2}}} \frac{2}{\epsilon} .
$$

\section{Box}

The diagram to be considered is shown in Fig. 4. The integral to be computed is

$$
\begin{aligned}
I_{B}\left(p, m_{0}, d\right) & =\frac{\mu_{B}}{4 !}\left(\frac{\lambda_{0}}{12}\right)^{2} A_{B}\left(p, k, l, m_{0}, d\right) \\
& =\frac{1}{6} \lambda_{0}^{2} A_{B}\left(p, k, l, m_{0}, d\right),
\end{aligned}
$$

where $\mu_{B}=244$ ! denotes the multiplicity of this contribution leading to the overall factor $\frac{1}{6}$. The quantity $A_{B}\left(p, k, l, m_{0}, d\right)$ is

$$
\begin{aligned}
A_{B}\left(p, k, l, m_{0}, d\right)= & \int \frac{d^{d} q}{(2 \pi)^{d}} \frac{1}{\left((k-q)^{2}+m_{0}^{2}\right)\left(q^{2}+M^{2}\right)^{\beta}} \\
& \times \frac{1}{\left((q+p)^{2}+m_{0}^{2}\right)\left((q+p-l)^{2}+M^{2}\right)^{\beta}} .
\end{aligned}
$$

The degree of divergence of this integral is

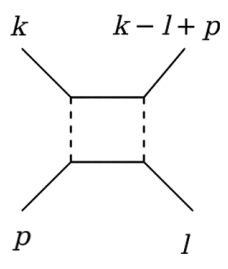

FIG. 4. Contributions to the 4 and 6 point four-point $\phi$ function. 


$$
\omega_{B}=d-4-4 \beta
$$

which, for the cases considered in this work $\left(d=n-\epsilon, \beta=\frac{n}{2}-2\right)$, is

$$
\omega_{B}=4-n-\epsilon .
$$

This shows that, for $n>4$, there is no divergence for $\epsilon \rightarrow 0$. Introducing the Feynman parametrization leads to

$$
A_{B}\left(p, k, l, m_{0}, d\right)=\int_{0}^{1} d x d y d z \frac{(1-x)^{\beta-1} y^{\beta-1}((1-z) z)^{\beta} \Gamma(2+2 \beta)}{(4 \pi)^{\frac{d}{2}} \Gamma(\beta)^{2}} \mu^{4-n-\epsilon} P(k, p, l, x, y, z),
$$

where

$$
\begin{aligned}
P(k, p, l, x, y, z)= & \int \frac{d^{d} q}{(2 \pi)^{d}}\left(( 1 - z ) \left((1-y)\left((k-q)^{2}+m_{0}^{2}\right)+\left((1-z)\left(y\left((q+p-l)^{2}+M^{2}\right)\right)\right)\right.\right. \\
& \left.+z\left(x\left((q+p)^{2}+m_{0}^{2}\right)-(x-1)\left(q+M^{2}\right)^{2}\right)\right)^{2-n}
\end{aligned}
$$

is positive definite in the $x, y, z$ integration region. The integral in the last expression can be done, leading to

$$
\begin{aligned}
P(k, p, l, x, y, z)= & \frac{\Gamma\left(-\frac{d}{2}+2 \beta+2\right)}{\Gamma(2+2 \beta)}\left(k^{2}(y-1)(z-1)-\left(k(y(1-z)+z-1)+M^{2}(1-x) z+p(x z+y(1-z))\right.\right. \\
& +q y z-q y)^{2}+M^{4}(1-x) z-M^{2} y z+M^{2} y+m_{0}^{2}((x-1) z+y(z-1)+1)+p^{2} x z+p^{2} y(1-z) \\
& \left.+2 p q y z-2 p q y+q^{2} y(1-z)\right)^{\frac{1}{2}(d-4(\beta+1))} .
\end{aligned}
$$

It is noted that, for $n \leq 4$, the power appearing in $P(k, p, l, x, y, z)$ is positive or goes to zero for $\epsilon \rightarrow 0$. In addition, $P(k, p, l, x, y, z)$ is well defined and positive in the $x, y, z$ integration region. Thus the integral in Eq. (4.4) is bounded from above by the integral of $P(k, p, l, x, y, z)$ multiplied by the following positive integral:

$$
\mu^{4-n-\epsilon} \int_{0}^{1} d x d y d z \frac{(1-x)^{\beta-1} y^{\beta-1}(-(z-1) z)^{\beta}}{(4 \pi)^{\frac{d}{2}} \Gamma(\beta)^{2}}=\frac{\mu^{4-n-\epsilon}}{(4 \pi)^{\frac{d}{2}} \Gamma(2 \beta+2)} \stackrel{d=n-\epsilon, \beta=\frac{n}{2}-2}{=} \frac{\mu^{4-n-\epsilon}}{(4 \pi)^{\frac{d}{2}} \Gamma(n-2)} .
$$

Therefore the singularities of $A_{B}\left(p, k, l, m_{0}, d\right)$ for $\epsilon \rightarrow 0$ arise only from the factor

$$
\frac{\Gamma\left(-\frac{d}{2}+2 \beta+2\right)}{\Gamma(n-2)}=\frac{\Gamma\left(\frac{n}{2}+\frac{\epsilon}{2}-2\right)}{\Gamma(n-2)} .
$$

This produces singularities only for $n=4$. For this value of $n$ and in the limit $\epsilon \rightarrow 0, P(k, p, l, x, y, z) / \Gamma\left(-\frac{d}{2}+2 \beta+2\right)$ can be replaced by 1 , leading to

$$
\begin{aligned}
A_{B}\left(m_{0}, n-\epsilon\right) & =\mu^{4-n-\epsilon} \int_{0}^{1} d x d y d z \frac{(1-x)^{\beta-1} y^{\beta-1}(-(z-1) z)^{\beta} \Gamma\left(-\frac{d}{2}+2 \beta+2\right)}{(4 \pi)^{\frac{d}{2}} \Gamma(\beta)^{2}} \\
& =\mu^{4-n-\epsilon} \frac{\Gamma\left(-\frac{d}{2}+2 \beta+2\right)}{(4 \pi)^{\frac{d}{2}} \Gamma(2 \beta+2)} \stackrel{d=n-\epsilon, \beta=\frac{n}{2}-2}{=} \mu^{4-n-\epsilon} \frac{\Gamma\left(\frac{n}{2}+\frac{\epsilon}{2}-2\right)}{(4 \pi)^{\frac{d}{2}} \Gamma(n-2)},
\end{aligned}
$$

which presents a pole term in $\epsilon$ only for $n=4$, given by

$$
A_{B}^{P T}\left(p, k, l, m_{0}, 4-\epsilon\right)=\frac{1}{8 \pi^{2} \epsilon} .
$$


Thus

$$
I_{B}^{P T}\left(p, m_{0}, d\right)=\frac{1}{6} \lambda_{0}^{2} \frac{\mu^{-\epsilon}}{(4 \pi)^{2}} \frac{2}{\epsilon} \delta_{n, 4} .
$$

\section{Effective quartic coupling and its $\beta$ function}

Summing up the contributions from the $\rho \phi^{2}$ vertex correction, the $\rho$ self-energy correction, and the box and including the field renormalization in Eq. (4.3) leads to

$$
\lambda=\frac{\lambda_{0} Z_{\phi}^{2}}{Z_{g}}
$$

where

$$
\begin{aligned}
& Z_{\phi}=1-\frac{g_{0}}{(4 \pi)^{\frac{n}{2}}} \frac{1}{\epsilon} c_{\phi}(n), \\
& Z_{g}=1+\frac{g_{0}}{(4 \pi)^{\frac{n}{2}}} \frac{1}{\epsilon} c_{g}(n),
\end{aligned}
$$

where

$$
c_{g}(n)=\frac{4}{3} \frac{1}{\Gamma\left(\frac{n}{2}\right)}+\delta_{n, 4} \frac{4}{3}+\delta_{n, 4} \frac{1}{3},
$$

the beta function corresponding to the adimensional coupling $g$, is

$$
\begin{aligned}
\beta(g) & =\mu \frac{d}{d \mu} g=\mu \frac{d}{d \mu}\left(\frac{\lambda_{0} Z_{\phi}^{2}}{Z_{g}} \mu^{-\epsilon}\right) \\
& =-\epsilon g+2 g Z_{\phi}^{-1} \mu \frac{d}{d \mu} Z_{\phi}-g Z_{g}^{-1} \mu \frac{d}{d \mu} Z_{g} \\
& =-\epsilon g+4 g \gamma-\frac{c_{g}(n)}{(4 \pi)^{\frac{n}{2}} \epsilon} g \beta(g),
\end{aligned}
$$

which leads to

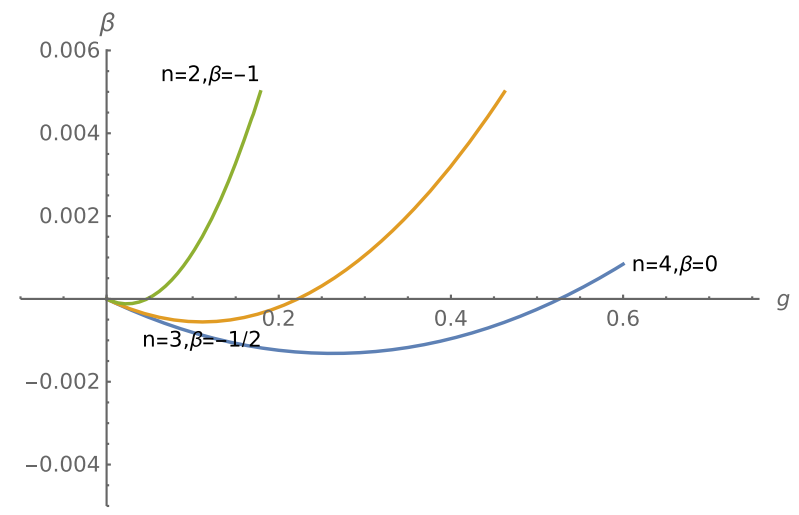

$$
\begin{aligned}
\beta(g) & =-\epsilon g+4 g \gamma+g^{2} \frac{c_{g}(n)}{(4 \pi)^{\frac{n}{2}}} \\
& =-\epsilon g+\frac{g^{2}}{(4 \pi)^{\frac{n}{2}}}\left(2 c_{\phi}(n)+c_{g}(n)\right) .
\end{aligned}
$$

The fixed points defined by $\beta\left(g^{\star}\right)=0$ are given by

$$
g_{0}^{\star}=0, \quad g_{1}^{\star}=\frac{\epsilon(4 \pi)^{\frac{n}{2}-\frac{e}{2}}}{2 c_{\phi}(n)+c_{g}(n)} .
$$

For $n=2,3,4,5, C(n)>0$, so that for $\epsilon>0, g_{1}^{\star}$ is positive and corresponds to an IR fixed point. Also, for $\epsilon>0, g_{0}^{\star}$ corresponds to an UV fixed point. The Fig. 5 show these $\beta$ functions.

\section{The critical exponents $\boldsymbol{\eta}$ and $\nu$}

The $\gamma_{m}(n)$ function is given by

$$
\begin{aligned}
\gamma_{m}(n) & =\gamma-\frac{c_{m^{2}}(n)}{(4 \pi)^{\frac{n}{2}}} \frac{\beta(g)}{2 \epsilon}=\frac{1}{2} \frac{g}{(4 \pi)^{\frac{n}{2}}} c_{\phi}(n)+\frac{c_{m^{2}}(n)}{(4 \pi)^{\frac{n}{2}}} \frac{g}{2} \\
& =\frac{1}{2} \frac{g}{(4 \pi)^{\frac{n}{2}}}\left(c_{\phi}(n)+c_{m^{2}}(n)\right),
\end{aligned}
$$

where Eq. (4.5) was employed. The critical exponents are related to the fixed point values $\gamma^{\star}$ and $\gamma_{m}^{\star}$ of the functions $\gamma$ and $\gamma_{m}$. They are given by

$$
\nu(n)=\frac{1}{2-2 \gamma_{m}^{\star}(n)}, \quad \eta(n)=2 \gamma^{\star}(n) .
$$

The nontrivial fixed point is given by

$$
g_{1}^{\star}(n)=\frac{\epsilon(4 \pi)^{\frac{n}{2}}}{2 c_{\phi}(n)+c_{g}(n)},
$$

and the fixed point values $\gamma_{m}^{\star}(n)$ and $\gamma^{\star}(n)$ are therefore given by

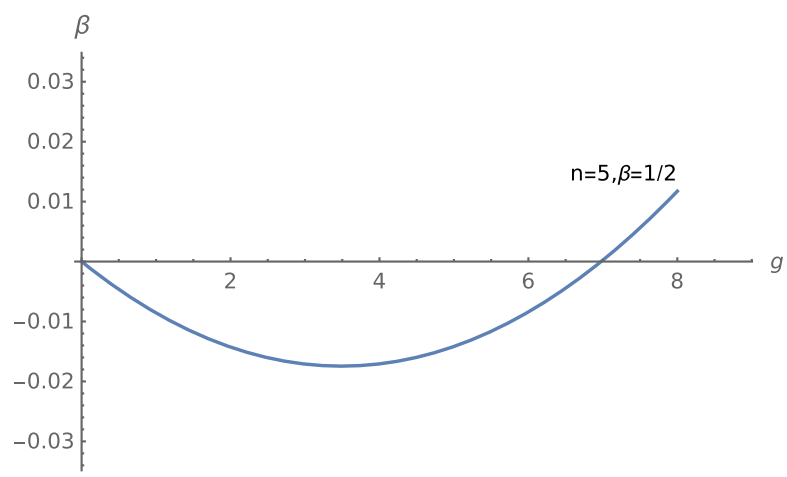

FIG. 5. Beta functions for $\epsilon=0.01$ and dimensions 2, 3, 4, and 5 . 


$$
\begin{aligned}
& \gamma_{m}^{\star}(n)=\frac{1}{2} \frac{g_{1}^{\star}(n)}{(4 \pi)^{\frac{n}{2}}}\left(c_{\phi}(n)+c_{m^{2}}(n)\right)=\frac{\epsilon}{2}\left(\frac{c_{\phi}(n)+c_{m^{2}}(n)}{2 c_{\phi}(n)+c_{g}(n)}\right), \\
& \gamma^{\star}(n)=\frac{1}{2} \frac{g_{1}^{\star}(n)}{(4 \pi)^{\frac{n}{2}}} c_{\phi}(n)=\frac{\epsilon}{2\left(2+\frac{c_{g}(n)}{c_{\phi}(n)}\right)} .
\end{aligned}
$$

This leads to

$$
\begin{gathered}
\gamma_{m}^{\star}(n)= \begin{cases}\frac{\epsilon}{4} & n=2 \\
\frac{\epsilon}{4} & n=3 \\
\frac{\epsilon}{6} & n=4, \\
\frac{1}{32} \epsilon\left(\frac{5 M^{2}}{\mathrm{~m} 0^{2}}+8\right) & n=5\end{cases} \\
\gamma^{\star}(n)= \begin{cases}\frac{\epsilon}{8} & n=2 \\
\frac{\epsilon}{16} & n=3 \\
0 & n=4 \\
-\frac{\epsilon}{16} & n=5\end{cases} \\
\nu(n)= \begin{cases}\frac{1}{2}+\frac{\epsilon}{8}+O\left(\epsilon^{2}\right) \\
\frac{1}{2}+\frac{\epsilon}{8}+O\left(\epsilon^{2}\right) \\
\frac{1}{2}+\frac{\epsilon}{12}+O\left(\epsilon^{2}\right) \\
\frac{1}{2}+\epsilon\left(\frac{5 M^{2}}{640^{2}}+\frac{1}{8}\right)+O\left(\epsilon^{2}\right) & n=5\end{cases} \\
\eta(n)= \begin{cases}\frac{\epsilon}{4} & n=2 \\
\frac{\epsilon}{8} & n=3 \\
0 & n=4 \\
-\frac{\epsilon}{8} & n=5\end{cases}
\end{gathered}
$$

It is noted that, for $n=5$, the critical exponent $\eta$ is negative. This violates the unitarity bound $\eta>0$, whose origin is explained in the next section.

\section{UNITARITY BOUNDS}

\section{A. The conformal algebra in $\boldsymbol{n}$ dimensions}

At the critical point, i.e., for $m=M=0$, the actions (2.1) and (2.2) are invariant under conformal transformations. It is assumed that there exist conserved charges implementing these transformations at the level of the quantum field. The conformal algebra for dimensions $n \geq 3$ is given by

$$
\begin{aligned}
{\left[D, P_{\mu}\right] } & =i P_{\mu}, \\
{\left[P_{\rho}, L_{\mu \nu}\right] } & =i\left(\eta_{\rho \mu} P_{\nu}-\eta_{\rho \nu} P_{\mu}\right), \\
{\left[D, K_{\mu}\right] } & =-i K_{\mu}, \\
{\left[K_{\mu}, P_{\nu}\right] } & =2 i\left(\eta_{\mu \nu} D-L_{\mu \nu}\right), \\
{\left[K_{\rho}, L_{\mu \nu}\right] } & =i\left(\eta_{\rho \mu} K_{\nu}-\eta_{\rho \nu} K_{\mu}\right), \\
{\left[L_{\mu \nu}, L_{\rho \sigma}\right] } & =i\left(\eta_{\nu \rho} L_{\mu \sigma}+\eta_{\mu \sigma} L_{\nu \rho}-\eta_{\mu \rho} L_{\nu \sigma}-\eta_{\nu \sigma} L_{\mu \rho}\right),
\end{aligned}
$$

where $P_{\mu}$ are the generators of translations, $L_{\mu \nu}$ the generators of rotations in the $\mu-\nu$ plane, $D$ the generator of dilatations, and $K_{\mu}$ the generators of special conformal transformations. In cylindrical coordinates, the hermiticity properties of operators are such that [12]

$$
P_{\mu}^{\dagger}=K_{\mu} .
$$

\section{B. Positive definite inner products and bounds for $\boldsymbol{\eta}$}

For a spinless primary state $|\Delta\rangle$, the commutation relation between $P_{\mu}$ and $K_{\nu}$ can be used to show that

$$
\begin{gathered}
\left.\left|P_{\mu}\right| \Delta\right\rangle\left.\right|^{2}>0 \Rightarrow \Delta>0, \\
\left.\left|P_{\mu} P_{\nu}\right| \Delta\right\rangle\left.\right|^{2}>0 \Rightarrow \Delta>\frac{n-2}{2}
\end{gathered}
$$

for a space of dimension $n$. Taking into account the oneloop corrections computed in the previous sections, the dimension of the field is

$$
[\phi]=\frac{n-2}{2}+\frac{\eta}{2} ;
$$

thus the unitarity bound implies

$$
\frac{\eta}{2}>0
$$

This is violated for $n \geq 5$.

This violation could be avoided by taking $\epsilon<0$ for $n=5$, but that would make the nontrivial fixed point $g_{1}^{\star}$ negative, which corresponds to an unstable theory. The same situation happens for any $n \geq 5$. This is a consequence of the sign change mentioned at the end of Sec. III A. This can also be traced back to the appearance of inverse powers of the Laplacian in the interaction term $(\beta>0)$.

\section{CONCLUSIONS AND OUTLOOK}

Conclusions and further research motivated by this work are summarized in the series of remarks given below:

(a) The critical behavior of a nonlocal scalar field theory is studied. This theory has a nonlocal quartic interaction term which involves a real power $-\beta$ of the Laplacian. The parameter $\beta$ can be tuned so as to make that interaction marginal for any dimension.

(b) As explained in Sec. III, only local counterterms in the fields $\rho$ and $\phi$ are required to renormalize this theory. In this respect, it is stressed that, in spite of the integer or half-integer powers of the Laplacian that appear in the action (2.2), the degree of divergence of any diagram is given by an integer. This fact is the crucial one that allows one to renormalize the theory employing only local counterterms in the fields, which are described in the Appendix. This property is not exclusive to this particular model. It would interesting 
to study the renormalization of other models involving these kind of "mild" nonlocalities.

(c) From the Wilson renormalization group point of view, there is no restriction on the range of interactions. Therefore it makes sense to study the renormalization of a nonlocal field theory. In this work, this has been done using the field theoretic version of this procedure. This was done for the first corrections to the two- and four-point functions. It was shown that the resulting critical theories are consistent, and for dimensions $d<5$, they give a unitary representation of the conformal group.

(d) For dimensions $d \geq 5$, the theories have a critical index $\eta<0$ which implies a violation of the unitarity bounds. These unitarity bounds are a consequence of requiring that the theory gives a unitary representation of the conformal group. Of course, the fact that this requirement is not fulfilled by these theories does not mean that they cannot be used to describe the physics of statistical models.

(e) It would be interesting to identify concrete statistical models whose criticality is described by these fixed points and critical exponents.

Summarizing, it is believed that the study of nonlocal field theories can enlarge our knowledge about the fixed points and renormalization group flows in the space of all possible couplings mentioned in the Introduction. In particular, the study presented in this work shows the existence of new nontrivial fixed points.

\section{ACKNOWLEDGMENTS}

I am deeply indebted to G. Torroba for sharing his expertise on the renormalization group, and for the many enlightening discussions. I also thank M. Solis Benitez for the many valuable discussions on the contents of this paper. The financial support provided by CONICET is gratefully acknowledged.

\section{APPENDIX: COUNTERTERMS}

When looking for an effective field theory at large distances, the important coupling constants are those related to relevant and marginal operators. These correspond to coupling constants with positive or vanishing dimensions in units of mass. ${ }^{4}$ The structure of the coupling constants that change under the scaling of distances and could survive in the infrared depends on $n$, i.e., on the dimension of space. ${ }^{5}$ A list of the terms $T$ in the Lagrangian corresponding to these coupling constants for each value of $n$ is given below:

\footnotetext{
${ }^{4}$ In field theoretic terms, these are the interactions corresponding, respectively, to super-renormalizable and renormalizable field theories.

${ }^{5}$ In field theoretic terms, these coupling constants correspond to the counterterms required to make the effective action finite.
}

(a) $n=4$. This case corresponds to the usual local $\phi^{4}$ field theory. In the table below, the degree of divergence $\omega=4-E_{\phi}-2 E_{\rho}$ and the corresponding terms are given as a function of the numbers $E_{\rho}, E_{\phi}$ of external $\rho$ and $\phi$ lines, respectively,

$\begin{array}{cccccc}E_{\rho} & 0 & 0 & 1 & 1 & 2 \\ E_{\phi} & 2 & 4 & 0 & 2 & 0 \\ \omega & 2 & 0 & 2 & 0 & 0 \\ T & \phi^{2},(\partial \phi)^{2} & \phi^{4} & \rho & \rho \phi^{2} & \rho^{2}\end{array}$.

To one-loop order, all of the coupling constants corresponding to these terms change under scaling except for the second one; the kinetic term $(\partial \phi)^{2}$ for the $\phi$ field does not change at one-loop order ${ }^{6}$ under scaling. In this case and in all cases appearing below, the one-point function for the $\rho$ field gives a momentum independent contribution to the expectation value of this field. These contributions can always be absorbed by means of a field $\rho$ redefinition.

(b) $n=5, \omega=5-\frac{3}{2} E_{\phi}-2 E_{\rho}$ :

$\begin{array}{ccccc}E_{\rho} & 0 & 1 & 1 & 2 \\ E_{\phi} & 2 & 0 & 2 & 0 \\ \omega & 2 & 3 & 0 & 1 \\ T & \phi^{2},(\partial \phi)^{2} & \rho & \rho \phi^{2} & \rho^{2}\end{array}$.

To one-loop order, all of the coupling constants corresponding to these terms change under scaling.

(c) $n=6, \omega=6-2 E_{\phi}-2 E_{\rho}$ :

$\begin{array}{cccccc}E_{\rho} & 0 & 1 & 1 & 2 & 3 \\ E_{\phi} & 2 & 0 & 2 & 0 & 0 \\ \omega & 2 & 4 & 0 & 2 & 0 \\ T & \phi^{2},(\partial \phi)^{2} & \rho & \rho \phi^{2} & \rho^{2},(\partial \rho)^{2} & \rho^{3}\end{array}$.

In contrast to the previous cases, the term $\rho^{3}$, which was not in the original Lagrangian, appears. Recalling that $[\rho]=2$, it is noted that the dimension of this term is 6, which implies that the corresponding coupling constant is dimensionless, therefore corresponding to a marginal term. This term should therefore be included in the starting Lagrangian, which can contribute at one-loop order to the scaling of other couplings. For example, the diagram in Fig. 6 can, in principle, contribute to the $\phi^{4}$ term.

This is a general situation for the cases $n>5$. Additional terms which were not in the original Lagrangian are generated at longer distances. These terms include additional kinetic terms for the field $\rho$ with higher powers of the Laplacian and powers of the field $\rho$. All of these terms

\footnotetext{
${ }^{6}$ There is no field renormalization at one-loop order for the $\phi^{4}$ field theory in four dimensions.
} 


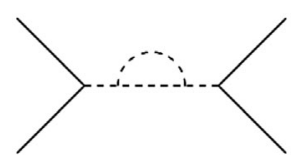

FIG. 6. Contribution to the four-point $\phi$ function originating from the $\rho^{3}$ term.

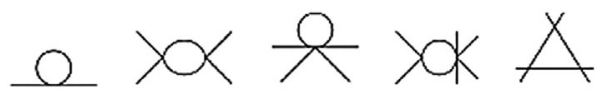

FIG. 7. Contributions to the four- and six-point $\phi$ functions originating from the $\phi^{4}$ and $\phi^{6}$ terms which vanish for $n=3$.

correspond to relevant or marginal couplings and the number of them is finite. This is so because, as shown in the table above and as argued in the previous subsection, the theory is renormalizable. In other words, it is a fact for these theories that the number of divergent types of diagrams does not increase with the perturbative order. Although this is not done in this work, it is noted that the inclusion of these terms presents, apart from the computation of additional diagrams, no conceptual difficulty. In this respect, it is worth noting that when going to higher dimensions, nontrivial coupling mixing can appear. For example, at $n=8$, apart from the kinetic terms, there are four couplings corresponding to interacting terms of dimension 8 that could, in principle, mix among themselves. This situation is similar to the one that appears in the models considered in [11].

(d) $n=3, \omega=3-\frac{1}{2} E_{\phi}-2 E_{\rho}$ :

$$
\begin{array}{cccccc}
E_{\rho} & 0 & 0 & 0 & 1 & 1 \\
E_{\phi} & 2 & 4 & 6 & 0 & 2 \\
\omega & 2 & 1 & 0 & 1 & 0 \\
T & \phi^{2},(\partial \phi)^{2} & \phi^{4} & \phi^{6} & \rho & \rho \phi^{2}
\end{array}
$$

In this case, the additional terms $\phi^{4}$ and $\phi^{6}$ appear. Neither vertex appears in the singular contribution to one-loop order. In Fig. 7, diagrams that involve these vertices and could, in principle, contribute at one-loop order are shown. However, they do not contribute because the self-contraction diagram and the fish diagram, shown in Fig. 8, both converge for $n=3$. The last diagram in Fig. 6 is also convergent for $n=3$.

(e) $n=2, \omega=2-2 E_{\rho}$ :

$\begin{array}{ccc}E_{\rho} & 0 & 1 \\ E_{\phi} & N & N^{\prime} \\ \omega & 2 & 0 \\ T & \phi^{N} & \rho \phi^{N^{\prime}}\end{array}$.

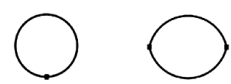

FIG. 8. Self-contraction and fish diagrams.

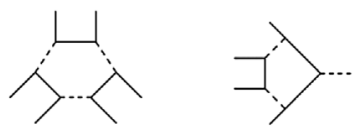

FIG. 9. Diagrams that vanish for $n=2$.

This case is rather peculiar. There are infinite terms with marginal couplings; these correspond to all possible positive even integer values for $N$ and $N^{\prime}$ above ( $N^{\prime}$ can also be zero). This is so because the $\phi$ field is dimensionless for $n=2$. This situation also appears for the usual local scalar theory at $n=2$, which is the same theory as the one considered here but with $\beta=0$ and $n=2$. In that case, all of the divergent diagrams can be made finite by normal ordering (see Appendix A10.2 of [13]). In the present case, however, the situation is more involved because the Laplacian appearing in the interaction term $\phi^{2}(-\triangle) \phi^{2}$ introduces momentum, equivalently, because the $\rho$ field has a nontrivial "kinetic" term. No general results for this model are presented in this work. However, in some specific examples appearing in Fig. 9, a situation similar to the one of the box diagram arises for these other diagrams. That is, an explicit calculation of these diagrams shows that the contributions are proportional to a quotient of gamma functions of the form

$$
\frac{\Gamma(a(\epsilon))}{\Gamma(j+j \beta)},
$$

where $j$ is a positive integer and $a(\epsilon)$ is positive and finite for $\epsilon \neq 0$. Recalling that $\beta=-1$ for $n=2$ shows that these diagrams give vanishing contributions because the gamma function in the denominator diverges when $\beta=-1$. The key ingredient producing this vanishing is the fact that, in all of these diagrams, the propagators for the $\phi$ field and the $\rho$ field can be paired, giving a power of momenta squared that is $-(1+\beta)$. Other diagrams contributing to the terms with $\left(E_{\rho}=0\right.$, $\left.E_{\phi}=N\right)$ and $\left(E_{\rho}=1, E_{\phi}=N^{\prime}\right)$ either converge or have the one-loop diagrams as subdiagrams. Therefore it is expected that only the cases $\left(E_{\rho}=0, E_{\phi}=2\right)$ and $\left(E_{\rho}=1, E_{\phi}=0\right.$ or 2$)$ get nonvanishing contributions. It would be interesting to have a general proof or a counterexample of this assertion. It would also be interesting to consider this theory from the point of view of conformal field theories. 
[1] K. G. Wilson and M. E. Fisher, Critical Exponents in 3.99 Dimensions, Phys. Rev. Lett. 28, 240 (1972).

[2] K. G. Wilson and J.B. Kogut, The renormalization group and the epsilon expansion, Phys. Rep. 12, 75 (1974).

[3] See, for example, A. O. Barvinsky, Aspects of nonlocality in quantum field theory, quantum gravity and cosmology, Mod. Phys. Lett. A 30, 1540003 (2015).

[4] See, for example, and D. N. Blaschke, Aspects of perturbative quantum field theory on non-commutative spaces, Proc. Sci., CORFU2015 (2016) 104.

[5] V.A. Fateev and A. B. Zamolodchikov, Parafermionic currents in the two-dimensional conformal quantum field theory and self-dual critical points in $Z_{N}$ invariant statistical systems, Zh. Eksp. Teor. Fiz. 89, 380 (1985) [Sov. Phys. JETP 62, 215 (1985)].

[6] A. S. Reddy, Nonlocal field theories at finite temperature and density, Ph.D. thesis, University of Minnesota, 2014.
[7] M. F. Paulos, S. Rychkov, B. C. van Rees, and B. Zan, Conformal invariance in the long-range Ising model, Nucl. Phys. B902, 246 (2016).

[8] P. W. Egolf and K. Hutter, The mean field theories of magnetism and turbulence, Entropy 19, 589 (2017).

[9] J. Honkonen and M. Y. Nalimov, Two-parameter expansion in the renormalization-group analysis of turbulence, Z. Phys. B 99, 297 (1995).

[10] R. Trinchero, Critical behavior of a nonlocal $\phi^{4}$ field theory and asymptotic freedom, Phys. Rev. D 98, 056023 (2018).

[11] M. Safari and G. P. Vacca, Uncovering novel phase structures in $\square^{k}$ scalar theories with the renormalization group, Eur. Phys. J. C 78, 251 (2018).

[12] J. D. Qualls, Lectures on conformal field theory, arXiv:1511 .04074

[13] J. Zinn-Justin, Quantum Field Theory and Critical Phenomena, 3rd ed., International Series of Monographs on Physics Vol. 113 (Clarendon Press, Oxford, 1996). 